\title{
Phenytoin versus levetiracetam as prophylaxis for postcraniotomy seizure in patients with no history of seizures: systematic review and meta-analysis
}

\author{
Chang-Hyun Lee, MD, MSc, ${ }^{1}$ Hae-Won Koo, MD, ${ }^{3}$ Seong Rok Han, MD, PhD, ${ }^{2}$ \\ Chan-Young Choi, MD, PhD, ${ }^{2}$ Moon-Jun Sohn, MD, PhD, ${ }^{3}$ and Chae-Heuck Lee, MD, PhD ${ }^{2}$
}

1Department of Neurosurgery, Seoul National University Hospital, Seoul; ${ }^{2}$ Department of Neurosurgery, Ilsan Paik Hospital, Inje University College of Medicine; and ${ }^{3}$ Department of Neurosurgery, Neuroscience \& Radiosurgery Hybrid Research Center, Ilsan Paik Hospital, Inje University College of Medicine, Goyang, Republic of Korea

OBJECTIVE De novo seizure following craniotomy (DSC) for nontraumatic pathology may adversely affect medical and neurological outcomes in patients with no history of seizures who have undergone craniotomies. Antiepileptic drugs (AEDs) are commonly used prophylactically in patients undergoing craniotomy; however, evidence supporting this practice is limited and mixed. The authors aimed to collate the available evidence on the efficacy and tolerability of levetiracetam monotherapy and compare it with that of the classic AED, phenytoin, for DSC.

METHODS PubMed, Embase, Web of Science, and the Cochrane Library were searched for studies that compared levetiracetam with phenytoin for DSC prevention. Inclusion criteria were adult patients with no history of epilepsy who underwent craniotomy with prophylactic usage of phenytoin, a comparator group with levetiracetam treatment as the main treatment difference between the two groups, and availability of data on the numbers of patients and seizures for each group. Patients with brain injury and previous seizure history were excluded. DSC occurrence and adverse drug reaction $(A D R)$ were evaluated. Seizure occurrence was calculated using the Peto odds ratio (POR), which is the relative effect estimation method of choice for binary data with rare events.

RESULTS Data from 7 studies involving 803 patients were included. The DSC occurrence rate was 1.26\% (4/318) in the levetiracetam cohort and $6.60 \%$ (32/485) in the phenytoin cohort. Meta-analysis showed that levetiracetam is significantly superior to phenytoin for DSC prevention (POR 0.233, 95\% confidence interval [Cl] 0.117-0.462, p<0.001). Subgroup analysis demonstrated that levetiracetam is superior to phenytoin for DSC due to all brain diseases (POR 0.129, 95\% $\mathrm{Cl} 0.039-0.423, p=0.001$ ) and tumor (POR 0.282, 95\% CI 0.117-0.678, $p=0.005$ ). ADRs in the levetiracetam group were cognitive disturbance, thrombophlebitis, irritability, lethargy, tiredness, and asthenia, whereas rash, anaphylaxis, arrhythmia, and hyponatremia were more common in the phenytoin group. The overall occurrence of ADR in the phenytoin $(34 / 466)$ and levetiracetam (26/432) groups $(p=0.44)$ demonstrated no statistically significant difference in ADR occurrence. However, the discontinuation rate of AEDs due to ADR was 53/297 in the phenytoin group and 6/196 in the levetiracetam group (POR 0.266, 95\% Cl 0.137-0.518, $\mathrm{p}<0.001$ ).

CONCLUSIONS Levetiracetam is superior to phenytoin for DSC prevention for nontraumatic pathology and has fewer serious ADRs that lead to discontinuation. Further high-quality studies that compare levetiracetam with placebo are necessary to provide evidence for establishing AED guidelines.

https://thejns.org/doi/abs/10.3171/2018.4.JNS1891

KEYWORDS levetiracetam; phenytoin; seizure; de novo; craniotomy; brain tumor; epilepsy

ABBREVIATIONS ADR = adverse drug reaction; $\mathrm{AED}=$ antiepileptic drug; $\mathrm{Cl}=$ confidence interval; $\mathrm{DSC}=$ de novo seizure following craniotomy; $\mathrm{POR}=\mathrm{Peto}$ odds ratio; $\mathrm{RCT}=$ randomized controlled trial.

SUBMITTED January 11, 2018. ACCEPTED April 5, 2018.

INCLUDE WHEN CITING Published online July 13, 2018; DOI: 10.3171/2018.4.JNS1891. 
$\mathrm{T}$ HE occurrence of de novo seizures following supratentorial craniotomy for nontraumatic pathology is estimated to be between $15 \%$ and $20 \%$, potentially resulting in debilitating medical, neurological, and psychosocial effects. ${ }^{9}$ However, the actual risk of de novo seizure following craniotomy (DSC) may vary from $3 \%$ to $92 \%$ over a 5 -year period because of the nature of the underlying disease for which surgery is undertaken. ${ }^{34}$ Three similar surveys published in 2003, 2005, and 2016 revealed that approximately $70 \%$ of polled neurosurgeons acknowledged routinely administering antiepileptic drugs (AEDs) pre- or postoperatively to prevent seizures following craniotomy. ${ }^{4,6,28}$

A randomized trial of postcraniotomy seizure revealed that phenytoin did not decrease seizure occurrence and that routine phenytoin administration was associated with significant drug-related morbidity. ${ }^{35}$ In 2000, the American Academy of Neurology released a statement against the routine use of AEDs in patients with brain tumor without a seizure history. ${ }^{11}$ However, many researchers concluded that these findings did not meet clear clinical decision-making criteria to support AED administration for DSC prevention. ${ }^{27,31,32,34}$ In the past, the most commonly used AEDs for prophylaxis against perioperative seizure were phenytoin, carbamazepine, and valproate, ${ }^{20}$ which were reported to be ineffective according to the results of a previous meta-analysis. ${ }^{30}$ In light of these conflicting studies, older ineffective AEDs have been replaced by newer, more efficacious ones as the preferred prophylactic AEDs for DSC. A recent survey showed that the primary AED for DSC prophylaxis has changed from phenytoin in 2005 to levetiracetam in 2016; levetiracetam was the primary agent used by $85 \%$ of AANS/CNS members, with only $14 \%$ of them using phenytoin. ${ }^{6}$ Although its mechanism of action is still not fully elucidated, levetiracetam appears to be a potent anticonvulsant drug. ${ }^{17}$ Levetiracetam has many advantages over classic AEDs; however, limited evidence is available to support levetiracetam's superiority in terms of efficacy. 7,25

The primary aim of the current study was to collate the available evidence on the efficacy and tolerability of levetiracetam monotherapy for DSC and compare it with that of a classic AED, phenytoin. To achieve this, we performed a systematic review of the literature, with a metaanalysis of relevant clinical studies.

\section{Methods}

\section{Search Strategy and Selection Criteria}

In accordance with the PRISMA (Preferred Reporting Items for Systematic Reviews and Meta-Analyses) guidelines, we performed a meta-analysis of clinical studies that investigated the DSC rate after prophylactic phenytoin or levetiracetam usage. We searched PubMed, Embase, Web of Science, and the Cochrane Database from inception to October 1, 2017, using the terms "levetiracetam" AND "seizure" AND "prophyla*" and their synonyms. There were no language restrictions on study eligibility, and only the largest study was included in the presence of overlapping study populations. In addition, the reference lists of reviews were screened for qualifying studies. Two inde- pendent researchers (coauthors on this study) performed separate literature searches. Any discrepancies among reviewers were solved through consensus and discussion with the corresponding author.

\section{Inclusion Criteria, Data Extraction, Endpoints, and Definitions}

The goals of the search were to find articles that met the following inclusion criteria: described a group of adult patients ( $\geq 18$ years of age) with no history of seizures who were undergoing craniotomy with prophylactic usage of phenytoin; described another group treated with levetiracetam, which was the main treatment difference between the two groups; and reported the number of patients and number of seizures for each group. Experimental studies, noncomparative studies, and case reports were excluded. We also excluded studies that included patients with brain injury, history of seizure disorders, and no prophylactic usage after craniotomy, as well as those that evaluated other AEDs.

We created a meta-analysis database of the selected studies with the following categories: study author, year of publication, study design, region, the rate of early ( $\leq$ 7 days) and late ( $>7$ days) seizures, patient age, number of seizures, AED used, AED dose, length of follow-up, and any complications related to the use of AEDs. Study quality was determined for controlled observational cohort studies with the risk of bias assessment tool for nonrandomized studies ${ }^{22}$ and for randomized controlled trials (RCTs) with the risk of bias tool.

\section{Statistical Analysis}

For each study, the number of seizures in patients treated with phenytoin or levetiracetam was identified, and the Peto odds ratio (POR) was calculated. The calculation of relative measures such as risk ratio and odds ratio necessitated the use of additional approaches for the case of zero events. In the case of zero events in one treatment arm, the POR can be calculated without continuity correction, and is currently the relative effect estimation method of choice for binary data with rare events. ${ }^{3}$

Heterogeneity was assessed using the Cochrane Q and $\mathrm{I}^{2}$ statistics. Heterogeneity was considered significant when the $\mathrm{p}$ value derived from Cochran $\mathrm{Q}$ was $<0.1$ or $\mathrm{I}^{2}$ $>50 \%$. Random-effects models were used depending on study design and the heterogeneity of the studies included. We assessed publication bias by visually inspecting the funnel plots and by calculating the $p$ value (2-sided) for Egger's intercept. We analyzed data using Comprehensive Meta-Analysis software (version 3.3, Biostat, Inc.).

\section{Results}

\section{Study Selection}

The initial search identified 525 articles, of which 221 duplicated articles were excluded (Fig. 1). Among the 304 remaining articles, 84 case reports, review articles, letters, or technical notes were excluded. Fifty-six studies that investigated posttraumatic seizure prophylaxis and 13 studies with pediatric patients were also excluded. The remaining 151 studies were subjected to a full-text review 


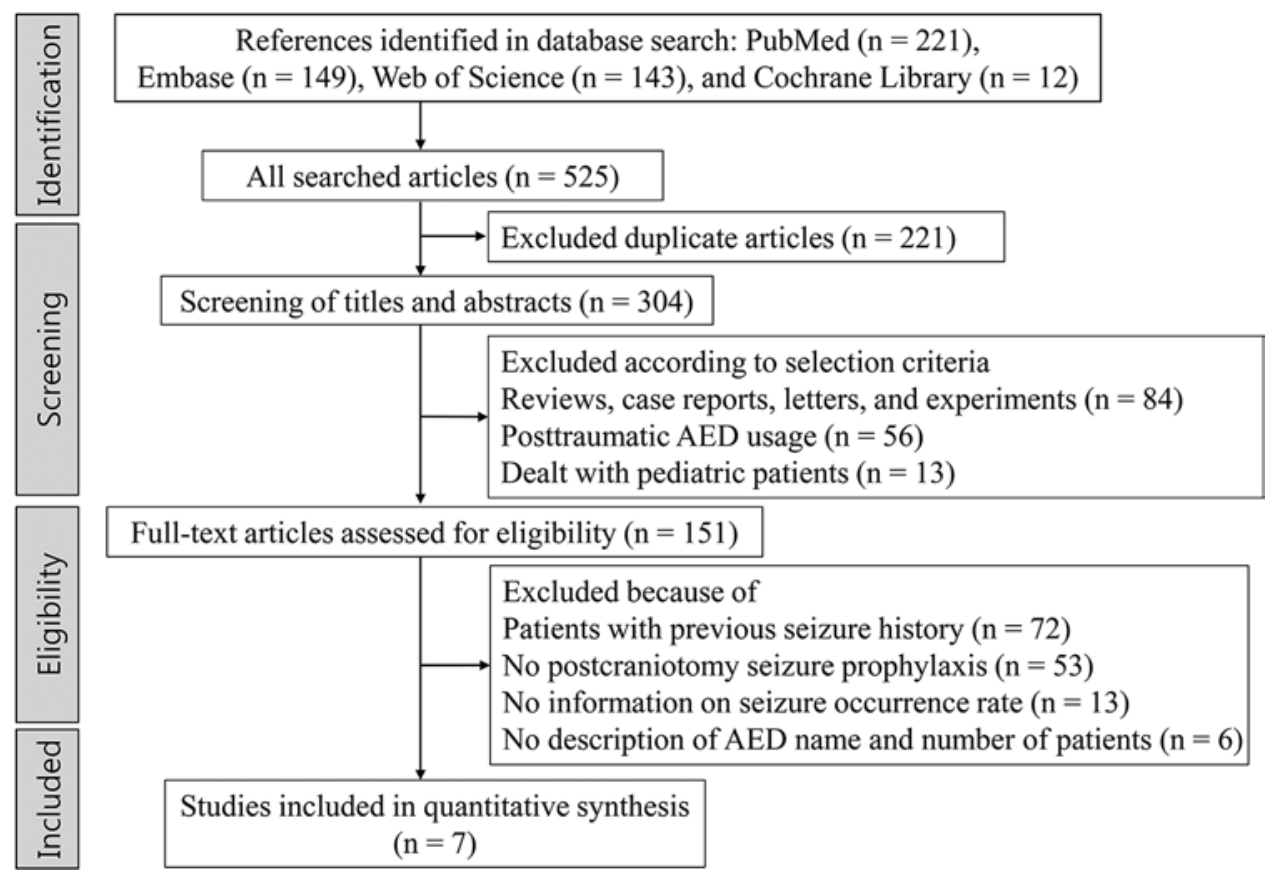

FIG. 1. Flow diagram for the selection of relevant studies.

and another 144 were excluded. The reasons for exclusion of these studies were previous seizure history $(n=72)$, no postcraniotomy seizure prophylaxis $(\mathrm{n}=53)$, no information on seizure rate $(n=13)$, and no description of AED name and number of patients $(n=6)$. Finally, 7 studies were eligible for the final analysis. Table 1 summarizes the key information extracted from the 7 studies included in this analysis.

The studies by Milligan et al. ${ }^{20}$ and Iuchi et al. ${ }^{13}$ included some patients with a history of seizures. Because they described the detailed number of patients with and without a history of seizures, we included only those patients who did not have a history of seizures. Studies by Milligan et al..$^{20}$ and Fuller et al. ${ }^{10}$ included 7 of 237 and 11 of 74 patients with traumatic brain injury, respectively. Although DSC in brain injury could not be delineated clearly, these two studies were included in this meta-analysis because the number of patients was small. The age range of patients in the study by Kern et al. was $8-85$ years old, which means that some pediatric patients were enrolled. $.^{15} \mathrm{How}-$ ever, this study was included because both the mean and median age were in the 50s. Other items showed low risk of bias in each included study. For two studies, researchers had received an award or grant from UCB Pharma, which manufactures levetiracetam, but they declared no conflicts of interest for the study. ${ }^{10,20}$

\section{Outcome Comparisons}

A total of 803 patients in 7 studies was included. The rate of DSC occurrence was $1.26 \%$ (4 of 318) in the levetiracetam cohort and $6.60 \%$ (32 of 485) in the phenytoin cohort. The POR of all individual studies trended in the same direction, and 4 studies showed significant superiority of levetiracetam (Fig. 2). A meta-analysis comparing leveti-

TABLE 1. Characteristics of included trials

\begin{tabular}{|c|c|c|c|c|c|c|c|c|c|c|c|}
\hline \multirow[b]{2}{*}{ Authors \& Year } & \multirow[b]{2}{*}{$\begin{array}{l}\text { Study } \\
\text { Design }\end{array}$} & \multirow[b]{2}{*}{ Disease } & \multirow[b]{2}{*}{ Country } & \multicolumn{4}{|c|}{ Levetiracetam Cohort } & \multicolumn{4}{|c|}{ Phenytoin Cohort } \\
\hline & & & & $\begin{array}{l}\text { No. of } \\
\text { Pts }\end{array}$ & DSC & $\begin{array}{c}\text { Mean Age, } \\
\text { yrs (SD) }\end{array}$ & $\begin{array}{c}\text { Males } \\
(\%)\end{array}$ & $\begin{array}{c}\text { No. of } \\
\text { Pts }\end{array}$ & DSC & $\begin{array}{c}\text { Mean Age, } \\
\text { yrs (SD) }\end{array}$ & $\begin{array}{c}\text { Males } \\
(\%)\end{array}$ \\
\hline Milligan et al., 2008 & Retro & All & USA & 72 & 0 & $54.0(15.1)$ & 38.9 & 165 & 3 & $61(18.4)$ & 47.3 \\
\hline Kern et al., 2012 & Retro & Tumor & Germany & 81 & 2 & $54(18.9)$ & 21.0 & 154 & 7 & $54(19.1)$ & 72.7 \\
\hline Fuller et al., 2013 & RCT & All & Australia & 36 & 0 & $58(16.1)$ & 50.0 & 38 & 6 & $60(15.8)$ & 50.0 \\
\hline D'Souza et al., 2011 & Pros & All & Australia & 25 & 0 & ND & ND & 18 & 3 & ND & ND \\
\hline luchi et al., 2015 & RCT & Tumor & Japan & 52 & 1 & $57(17.1)$ & 67.1 & 58 & 8 & $60(18.1)$ & 60.3 \\
\hline Kemerdere et al., 2016 & Retro & UIA & Turkey & 12 & 1 & $52.8(10.6)$ & 25.0 & 11 & 1 & $54.4(7.7)$ & 27.3 \\
\hline Höhne et al., 2016 & Retro & Tumor & Italy & 40 & 0 & 56.0 (ND) & 52.5 & 41 & 4 & 50 (ND) & 63.4 \\
\hline Total & & & & 318 & 4 & & & 485 & 32 & & \\
\hline
\end{tabular}

$\mathrm{ND}=$ not described; Pros = prospective cohort study; Pts = patients; Retro = retrospective cohort study; UIA = unruptured intracranial aneurysm. 


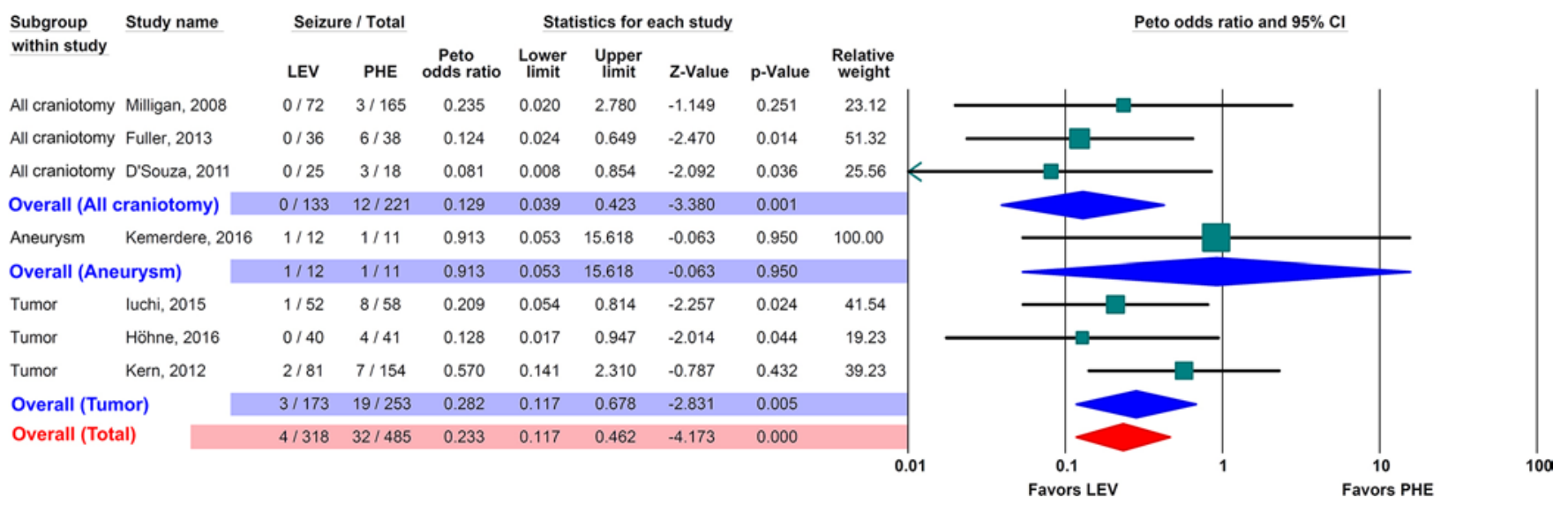

FIG. 2. Forest plots of occurrence of DSC by AED and disease. The overall POR was $0.233(95 \% \mathrm{Cl} 0.117-0.462)$, which indicates a significantly lower occurrence rate of DSC in the levetiracetam (LEV) cohort than in the phenytoin (PHE) cohort. Subgroup analysis by disease revealed a lower DSC rate in the levetiracetam cohort for patients with brain tumors and all brain diseases, but not patients with unruptured aneurysm. Figure is available in color online only.

racetam with phenytoin for the prevention of DSC showed that levetiracetam is significantly superior to phenytoin (POR 0.233, 95\% confidence interval [CI] 0.117-0.462, p $<0.001)$. We categorized the included studies according to disease as craniotomy performed for all brain diseases, cerebral unruptured aneurysm, and supratentorial tumor. Subgroup analysis by diseases demonstrated that levetiracetam was superior to phenytoin for occurrence of DSC in all brain disease (POR 0.129, 95\% CI 0.039-0.423, p $=0.001$ ) and tumor (POR 0.282, 95\% CI 0.117-0.678, p $=0.005)$ patients, but neither drug showed superiority for cerebral aneurysm (POR 0.913, 95\% CI 0.053-15.618, p $=0.950)$.

\section{Adverse Drug Reactions}

Three studies ${ }^{10,12,13}$ reported detailed adverse drug reactions (ADRs) and two studies ${ }^{8,20}$ reported only serious ADRs that lead to discontinuation of AEDs. The other two studies reported that there were no serious complications in either group. ${ }^{14,15}$ Overall occurrence of ADR was $34 / 466$ in the phenytoin group and 26/432 in the levetiracetam group $(\mathrm{p}=0.44)$, which demonstrated no statistically significant difference in ADR occurrence. However, the discontinuation rate of AEDs due to ADR was 53/297 in the phenytoin group and 6/196 in the levetiracetam group (POR 0.266, 95\% CI 0.137-0.518, p < 0.001), which demonstrated that the phenytoin cohort had a higher incidence of discontinuation of AEDs because of ADRs than the levetiracetam cohort (Fig. 3A). The detailed ADRs and their occurrence rates are shown in Table 2 and Fig. 3B. ADRs such as cognitive disturbance, thrombophlebitis, irritability, lethargy, tiredness, and asthenia were more frequent in the levetiracetam group, whereas rash, anaphylaxis, arrhythmia, and hyponatremia were more common in the phenytoin group.

\section{Sensitivity Analyses}

To determine if an individual study was responsible for the presence or absence of an effect in each of the statistical tests, we performed a series of sensitivity analyses regarding occurrence of seizures. These analyses showed no substantial difference after elimination of each single study (Fig. 4). There were insufficient data to perform a meta-analysis of detailed onset time of seizures. The funnel plot suggested an absence of significant publication bias within the studies (Fig. 5). The Egger test result for the comparison of interventions was $1.36(\mathrm{p}=0.13)$ for the intervention comparison. These results indicate that there was not substantial evidence of publication bias in the dataset.

\section{Discussion}

Neurosurgeons have long believed that preventing seizures with AEDs (seizure prophylaxis) is effective and necessary, but evidence supporting this practice is sparse and mixed. Our systematic review and meta-analysis provides strong evidence to support levetiracetam prophylaxis in adult patients with no history of seizures. Prophylactic levetiracetam usage for DSC was effective overall, and analysis of disease-specific efficacy revealed that it was beneficial in patients with supratentorial tumors. Discontinuation of phenytoin due to an ADR is much more frequent for phenytoin than levetiracetam. Thus, on the basis of these findings, levetiracetam is superior to phenytoin as a prophylactic AED for DSC with regard to both seizure control and ADR concerns.

A previous meta-analysis by Weston et al. failed to find a significant difference in seizure occurrence. ${ }^{34}$ However, our study indicated a significant difference in efficacy between levetiracetam and phenytoin. One presumed reason that the findings of Weston et al. were different from ours is that they included patients with a history of seizures. A previous multivariate analysis revealed that a preoperative history of seizures was a significant risk factor for DSC. ${ }^{1}$ Furthermore, neither the tumor type nor the type of surgery influenced seizure activity. ${ }^{30}$ Pourzitaki et al. reported a meta-analysis of prophylactic levetiracetam usage in patients with brain tumors; ${ }^{23}$ two of four studies included in the analysis investigated epilepsy patients with a his- 


\section{A. Forest plot of serious adverse drug reactions of antiepileptic drugs}

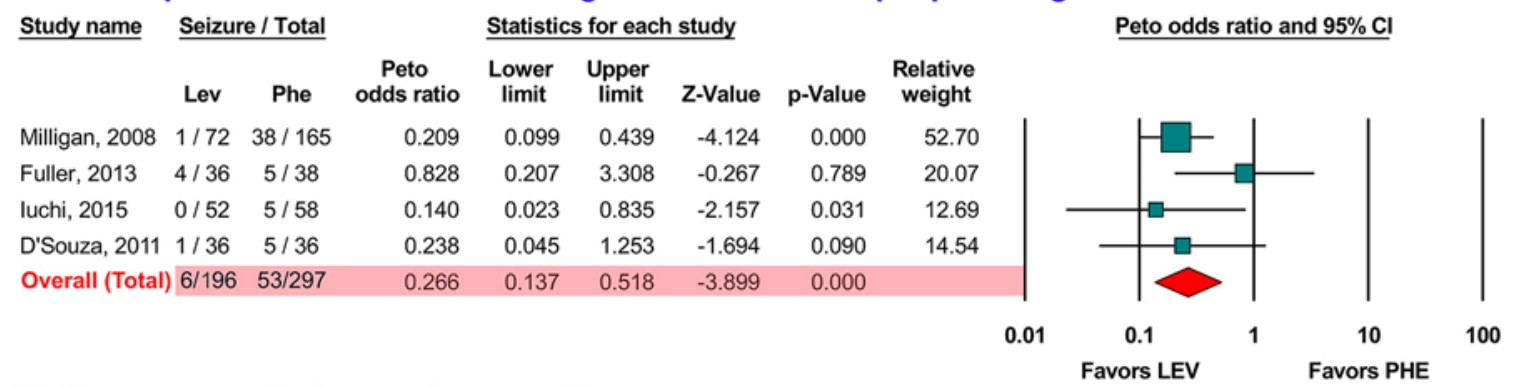

\section{B. Occurrence of adverse drug reactions}

Favors LEV Favors PHE

\section{Cognitive dysfunction}

Lethargy, tiredness, and asthenia

Irritability

Liver dysfunction
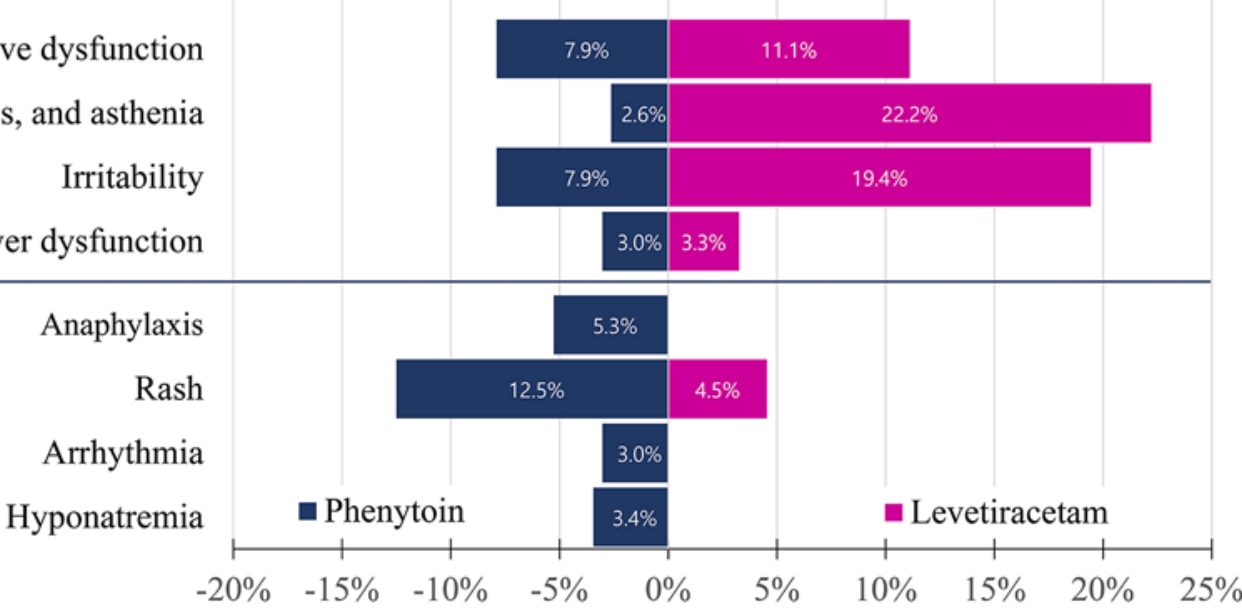

FIG. 3. A: Forest plot of serious ADRs that lead to discontinuation of AEDs. All studies show fewer incidences of serious ADRs in the levetiracetam group than in the phenytoin group. Discontinuation of AEDs was significantly more frequent in the phenytoin arm than the levetiracetam arm. Levetiracetam shows a superiority to phenytoin. B: Graph showing the occurrence of specific ADRs. Levetiracetam (purple) induced ADRs such as cognitive or psychological dysfunction, while phenytoin (navy) evoked allergic reaction, arrhythmia, and electrolyte imbalance. Figure is available in color online only.

tory of seizure disorders. Another meta-analysis by Yuan et al. also included studies and patients with and without seizure history. ${ }^{36}$

Another probable reason that previous studies failed to show the effectiveness of prophylactic AEDs was that they evaluated the use of phenytoin (a classic AED) for DSC prophylaxis, rather than newer AEDs such as levetiracetam. Some meta-analyses evaluating seizure prophylaxis included studies investigating monotherapy or combination therapy with phenytoin, phenobarbital, and valproic acid, and found no evidence to support DSC prophylaxis with any of these drugs or their combination. ${ }^{27,29,33}$ Our analysis used stricter inclusion criteria, excluding patients with epilepsy or head trauma, and added three recent direct comparative studies between phenytoin and levetiracetam published in 2015 and 2016. Even in this metaanalysis, a subgroup of cerebral aneurysm did not show a statistically significant difference in seizure prevention. Because only one study dealt with the subgroup, there is a limitation to interpretation and further studies are needed.

All 7 included studies in our analysis demonstrated a tendency of superiority of levetiracetam over phenytoin, and in total they showed statistically significant superiority of levetiracetam as a prophylactic AED for DSC. These results therefore demonstrate that our meta-analytical method is useful and effective. Although overall ADR oc- currence was not different between phenytoin and levetiracetam, serious ADRs necessitating discontinuation of the AED were significantly common in phenytoin. However, the data indicate that levetiracetam might induce ADRs such as cognitive or mood dysfunction, and caution is thus necessary in administering levetiracetam to patients with psychological diseases.

Although the mechanism of seizure prevention by levetiracetam is not yet fully understood, levetiracetam is a broad-spectrum AED that is effective against a variety of seizure types. ${ }^{5}$ Levetiracetam is a pyrridoline derivative with a very favorable pharmacokinetic profile, exhibiting excellent bioavailability, linear kinetics, minimal plasma protein binding, and quick achievement of steady-state concentrations. ${ }^{5,16}$ Moreover, many studies, including the present one, have reported significantly fewer ADRs in the levetiracetam cohort than in the phenytoin cohort when AED prophylaxis was given for supratentorial surgery. 5,10 , 12-14,20,26 Specific phenytoin and valproate characteristics, including nonlinear pharmacokinetics and alkaline formulation, ${ }^{18,19}$ mandate frequent monitoring, reduce tolerability, and limit the administration rate, thereby increasing the cost of care..$^{18}$ In contrast, levetiracetam presents a novel alternative AED with greater potency and a comparatively wide therapeutic window, not requiring slow titration or serological monitoring at standard doses..$^{10,24}$ 
TABLE 2. Adverse drug reactions by study

\begin{tabular}{lcc}
\hline \multicolumn{1}{c}{ Authors \& Year } & $\begin{array}{c}\text { Phenytoin } \\
\text { Group (\%) }\end{array}$ & $\begin{array}{c}\text { Levetiracetam } \\
\text { Group (\%) }\end{array}$ \\
\hline Fuller et al., 2013 & $3(8)$ & $4(11)$ \\
\hline Cognitive disturbance & $3(8)$ & $7(19)$ \\
\hline Irritability & $1(3)$ & $8(22)$ \\
\hline Lethargy, tiredness, \& asthenia & $2(5)$ & 0 \\
\hline Anaphylaxis & $5(13)$ & 0 \\
\hline Allergy, major rash & $5(13)$ & $4(11)$ \\
\hline Rash, itch & $3(8)$ & 0 \\
\hline Thrombophlebitis & $2(5)$ & 0 \\
\hline Drug intoxication & & 0 \\
\hline Höhne et al., 2016 & $1(3)$ & 0 \\
\hline Hepatotoxicity & $1(3)$ & \\
\hline Arrhythmia & & $3(6)$ \\
\hline luchi et al., 2015 & $2(3)$ & 0 \\
\hline Liver dysfunction & $2(3)$ & 0 \\
\hline Atrial fibrillation & $2(3)$ & 0 \\
\hline Hyponatremia & $2(3)$ & 0 \\
\hline Severe skin eruption & & 0 \\
\hline Kern et al., 2012 & 0 & \\
\hline "Severe side effects" & 0 & 0 \\
\hline Kemerdere et al., 2016 & & 0 \\
\hline "Serious complication" & & 0 \\
\hline
\end{tabular}

Because levetiracetam is metabolized mainly in the kidneys, it does not induce or interfere with CYP enzymes, and thus does not interact with chemotherapy. ${ }^{37}$ Moreover, levetiracetam enhances p53-mediated inhibition of $\mathrm{O}^{6}$-methylguanine-DNA methyltransferase and sensitizes glioblastoma cells to temozolomide; therefore, it is considered the AED of choice in patients with malignant gliomas. ${ }^{2}$ Indeed, some investigators have proposed that levetiracetam should be considered a first-line agent in patients with brain tumors. ${ }^{7,21}$

Some limitations of the present study need to be acknowledged and addressed. The first limitation concerns
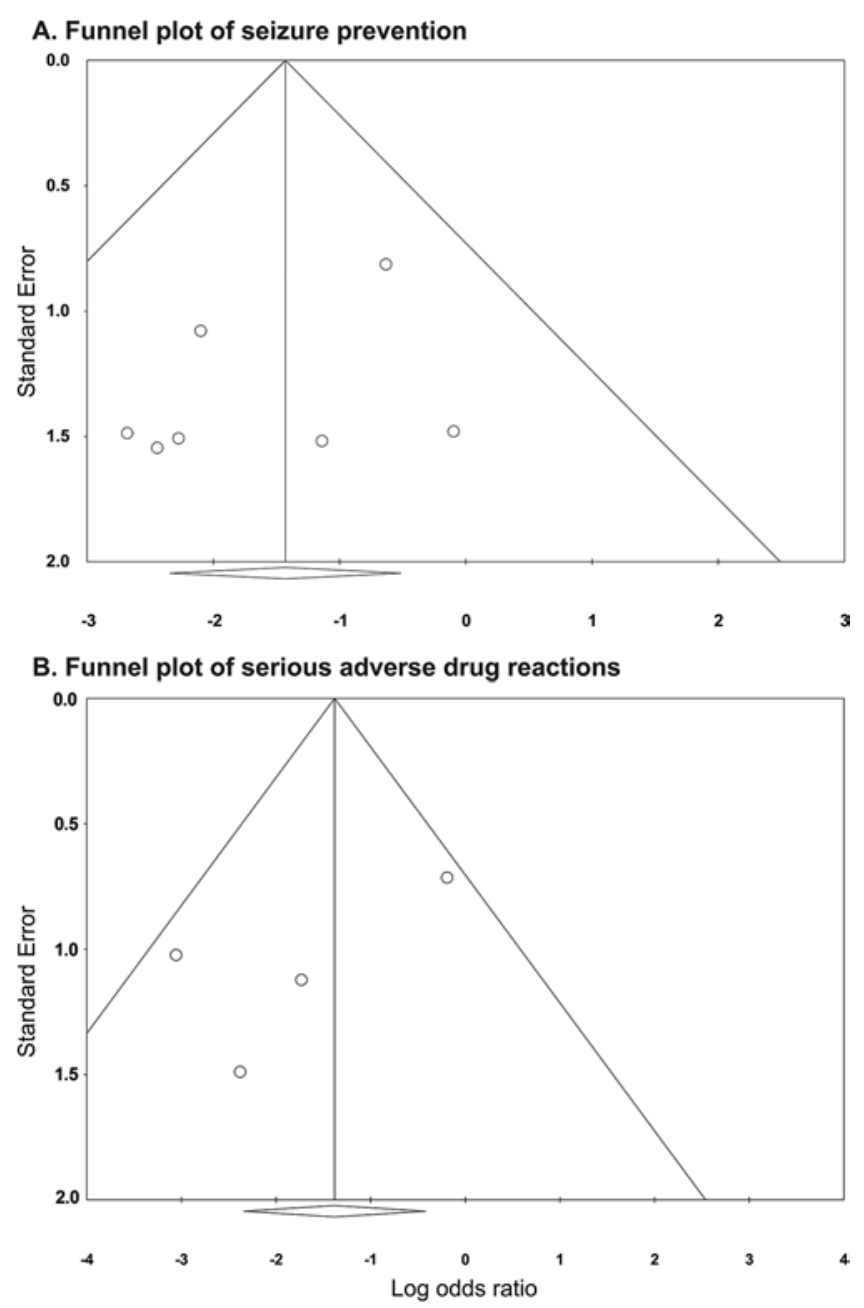

FIG. 5. Funnel plots regarding seizure prevention (A) and serious ADRs (B) showing symmetrical distribution of studies, indicating an absence of publication bias.

the heterogeneity of the included studies. A few patients with traumatic brain injury were included in this metaanalysis. However, they constituted a very small proportion of the total number of enrolled patients and likely did

\begin{tabular}{lccccc} 
Study name & \multicolumn{5}{c}{ Statistics with study removed } \\
& Point & $\begin{array}{c}\text { Lower } \\
\text { limit }\end{array}$ & $\begin{array}{c}\text { Upper } \\
\text { limit }\end{array}$ & Z-Value & p-Value \\
Milligan, 2008 & 0.232 & 0.089 & 0.609 & -2.968 & 0.003 \\
Fuller, 2013 & 0.275 & 0.105 & 0.722 & -2.620 & 0.009 \\
D'Souza, 2011 & 0.265 & 0.101 & 0.695 & -2.703 & 0.007 \\
Kemerdere, 2016 & 0.207 & 0.079 & 0.543 & -3.198 & 0.001 \\
luchi, 2015 & 0.280 & 0.101 & 0.774 & -2.454 & 0.014 \\
Höhne, 2016 & 0.262 & 0.100 & 0.688 & -2.721 & 0.007 \\
Kern, 2012 & 0.162 & 0.053 & 0.496 & -3.187 & 0.001 \\
& 0.240 & 0.096 & 0.599 & -3.054 & 0.002
\end{tabular}

FIG. 4. Sensitivity analysis via single elimination of each study regarding occurrence of DSC showed that the overall effect size did not change after elimination of any single study, indicating that no single study had a disproportionally large effect. Figure is available in color online only. 
not affect the results substantially. We controlled for key risk factors, such as history of seizures, and included only studies that performed a direct comparison of levetiracetam and phenytoin. Moreover, heterogeneity between included studies was low and acceptable. Second, the definition of seizure varied between the included studies, which used electroencephalograms, neurological signs, or both. Some investigators only recorded generalized seizures, and others recorded both partial and generalized seizures. Third, this meta-analysis showed that levetiracetam is superior to phenytoin, but not to placebo. There were few data comparing levetiracetam to placebo. Thus, there is no evidence of the superiority of levetiracetam over placebo. Finally, two included studies did not address serious complications regarding ADRs in either group. Because two studies did not report complications, underestimation of ADRs may be a reasonable explanation. Therefore, we excluded the two studies for analysis of ADRs. While this exclusion is contrary to the principle of meta-analysis that all data should be included, we believed this method was more reasonable in light of these considerations.

\section{Conclusions}

Our results indicate that, in patients with no history of seizures and nontraumatic pathology, levetiracetam is superior to phenytoin for DSC prevention. Levetiracetam is also advantageous in that it is associated with fewer serious ADRs that lead to discontinuation. Further high-quality, well-populated studies that compare levetiracetam with placebo are necessary to provide sufficient evidence for establishing AED guidelines for DSC prophylaxis.

\section{References}

1. Al-Dorzi HM, Alruwaita AA, Marae BO, Alraddadi BS, Tamim HM, Ferayan A, et al: Incidence, risk factors and outcomes of seizures occurring after craniotomy for primary brain tumor resection. Neurosciences (Riyadh) 22:107-113, 2017

2. Bobustuc GC, Baker CH, Limaye A, Jenkins WD, Pearl G, Avgeropoulos NG, et al: Levetiracetam enhances p53-mediated MGMT inhibition and sensitizes glioblastoma cells to temozolomide. Neuro Oncol 12:917-927, 2010

3. Brockhaus AC, Grouven U, Bender R: Performance of the Peto odds ratio compared to the usual odds ratio estimator in the case of rare events. Biom J 58:1428-1444, 2016

4. Brouwers MC, Chambers A, Perry J: Can surveying practitioners about their practices help identify priority clinical practice guideline topics? BMC Health Serv Res 3:23, 2003

5. Carreno M: Levetiracetam. Drugs Today (Barc) 43:769_ 794, 2007

6. Dewan MC, Thompson RC, Kalkanis SN, Barker FG II, Hadjipanayis CG: Prophylactic antiepileptic drug administration following brain tumor resection: results of a recent AANS/ CNS Section on Tumors survey. J Neurosurg 126:17721778,2017

7. Domínguez-Páez M, Herranz-Fernández JL, VillanuevaHaba V, Sánchez-Álvarez JC, Olivares-Granados G, Sola RG, et al: [Primary prophylaxis of early seizures after surgery of cerebral supratentorial tumors: Group for the Study of Functional-Sterotactic Neurosurgery of The Spain Society of Neurosurgery recommendations.] Neurocirugia (Astur) 23:29-35, 2012
8. D’Souza W, Fuller K, Murphy M, Cook MJ: Side effects and tolerability of IV levetiracetam vs. IV phenytoin and followon oral regimens in a neurosurgical patient population: a prospective randomised study. Epilepsy Curr 11 (Suppl 1): $1.273,2011$

9. Foy PM, Copeland GP, Shaw MD: The incidence of postoperative seizures. Acta Neurochir (Wien) 55:253-264, 1981

10. Fuller KL, Wang YY, Cook MJ, Murphy MA, D’Souza WJ: Tolerability, safety, and side effects of levetiracetam versus phenytoin in intravenous and total prophylactic regimen among craniotomy patients: a prospective randomized study. Epilepsia 54:45-57, 2013

11. Glantz MJ, Cole BF, Forsyth PA, Recht LD, Wen PY, Chamberlain $\mathrm{MC}$, et al: Practice parameter: anticonvulsant prophylaxis in patients with newly diagnosed brain tumors. Report of the Quality Standards Subcommittee of the American Academy of Neurology. Neurology 54:1886-1893, 2000

12. Höhne J, Schebesch KM, Ott C, Brawanski A, Lange M: The risk of hypotension and seizures in patients receiving prophylactic anti-epileptic drugs for supratentorial craniotomy. J Neurosurg Sci [epub ahead of print], 2016

13. Iuchi T, Kuwabara K, Matsumoto M, Kawasaki K, Hasegawa Y, Sakaida T: Levetiracetam versus phenytoin for seizure prophylaxis during and early after craniotomy for brain tumours: a phase II prospective, randomised study. J Neurol Neurosurg Psychiatry 86:1158-1162, 2015

14. Kemerdere R, Kacira T, Ak H, Ozyurt E, Tanriverdi T: Antiepileptic drug prophylaxis in unruptured intracranial aneurysms. Neurosurg Q 26:325-328, 2016

15. Kern K, Schebesch KM, Schlaier J, Hansen E, Feigl GC, Brawanski AT, et al: Levetiracetam compared to phenytoin for the prevention of postoperative seizures after craniotomy for intracranial tumours in patients without epilepsy. J Clin Neurosci 19:99-100, 2012

16. Lim DA, Tarapore P, Chang E, Burt M, Chakalian L, Barbaro N, et al: Safety and feasibility of switching from phenytoin to levetiracetam monotherapy for glioma-related seizure control following craniotomy: a randomized phase II pilot study. J Neurooncol 93:349-354, 2009

17. Löscher W, Brandt C: Prevention or modification of epileptogenesis after brain insults: experimental approaches and translational research. Pharmacol Rev 62:668-700, 2010

18. Martinelli EF, Mühlebach SF: Rapid i.v. loading with phenytoin with subsequent dose adaptation using non-steady-state serum levels and a Bayesian forecasting computer program to predict maintenance doses. J Clin Pharm Ther 28:385-393, 2003

19. Mattson RH: Parenteral antiepileptic/anticonvulsant drugs. Neurology 46 (6 Suppl 1):S8-S13, 1996

20. Milligan TA, Hurwitz S, Bromfield EB: Efficacy and tolerability of levetiracetam versus phenytoin after supratentorial neurosurgery. Neurology 71:665-669, 2008

21. Nasr ZG, Paravattil B, Wilby KJ: Levetiracetam for seizure prevention in brain tumor patients: a systematic review. $\mathbf{J}$ Neurooncol 129:1-13, 2016

22. Park J, Lee Y, Seo H, Jang B, Son H, Kim S: Risk of bias assessment tool for non-randomized studies (RoBANS): development and validation of a new instrument, in Proceedings of the 19th Cochrane Colloquium. London: Cochrane, 2011, pp 19-22

23. Pourzitaki C, Tsaousi G, Apostolidou E, Karakoulas K, Kouvelas D, Amaniti E: Efficacy and safety of prophylactic levetiracetam in supratentorial brain tumour surgery: a systematic review and meta-analysis. Br J Clin Pharmacol 82:315-325, 2016

24. Ramael S, Daoust A, Otoul C, Toublanc N, Troenaru M, Lu ZS, et al: Levetiracetam intravenous infusion: a randomized, placebo-controlled safety and pharmacokinetic study. Epilepsia 47:1128-1135, 2006 
25. Rudà R, Bello L, Duffau H, Soffietti R: Seizures in low-grade gliomas: natural history, pathogenesis, and outcome after treatments. Neuro Oncol 14 (Suppl 4):iv55-iv64, 2012

26. Rüegg S, Naegelin Y, Hardmeier M, Winkler DT, Marsch S, Fuhr P: Intravenous levetiracetam: treatment experience with the first 50 critically ill patients. Epilepsy Behav 12:477480,2008

27. Sayegh ET, Fakurnejad S, Oh T, Bloch O, Parsa AT: Anticonvulsant prophylaxis for brain tumor surgery: determining the current best available evidence. J Neurosurg 121:1139-1147, 2014

28. Siomin V, Angelov L, Li L, Vogelbaum MA: Results of a survey of neurosurgical practice patterns regarding the prophylactic use of anti-epilepsy drugs in patients with brain tumors. J Neurooncol 74:211-215, 2005

29. Sirven JI: Antiepileptic drug therapy for adults: when to initiate and how to choose. Mayo Clin Proc 77:1367-1375, 2002

30. Sirven JI, Wingerchuk DM, Drazkowski JF, Lyons MK, Zimmerman RS: Seizure prophylaxis in patients with brain tumors: a meta-analysis. Mayo Clin Proc 79:1489-1494, 2004

31. Skardelly M, Brendle E, Noell S, Behling F, Wuttke TV, Schittenhelm J, et al: Predictors of preoperative and early postoperative seizures in patients with intra-axial primary and metastatic brain tumors: a retrospective observational single center study. Ann Neurol 78:917-928, 2015

32. Temkin NR: Prophylactic anticonvulsants after neurosurgery. Epilepsy Curr 2:105-107, 2002

33. Tremont-Lukats IW, Ratilal BO, Armstrong T, Gilbert MR: Antiepileptic drugs for preventing seizures in people with brain tumors. Cochrane Database Syst Rev (2):CD004424, 2008

34. Weston J, Greenhalgh J, Marson AG: Antiepileptic drugs as prophylaxis for post-craniotomy seizures. Cochrane Database Syst Rev (3):CD007286, 2015
35. Wu AS, Trinh VT, Suki D, Graham S, Forman A, Weinberg JS, et al: A prospective randomized trial of perioperative seizure prophylaxis in patients with intraparenchymal brain tumors. J Neurosurg 118:873-883, 2013

36. Yuan Y, Yunhe M, Xiang W, Yanhui L, Yanwu Y, Shuang L, et al: P450 enzyme-inducing and non-enzyme-inducing antiepileptic drugs for seizure prophylaxis after glioma resection surgery: a meta-analysis. Seizure 23:616-621, 2014

37. Zachenhofer I, Donat M, Oberndorfer S, Roessler K: Perioperative levetiracetam for prevention of seizures in supratentorial brain tumor surgery. J Neurooncol 101:101-106, 2011

\section{Disclosures}

The authors report no conflict of interest concerning the materials or methods used in this study or the findings specified in this paper.

\section{Author Contributions}

Conception and design: Chang-Hyun Lee. Acquisition of data: Chang-Hyun Lee, Koo. Analysis and interpretation of data: Chang-Hyun Lee. Drafting the article: Chang-Hyun Lee. Critically revising the article: Choi. Reviewed submitted version of manuscript: all authors. Approved the final version of the manuscript on behalf of all authors: Chang-Hyun Lee. Statistical analysis: Chang-Hyun Lee. Administrative/technical/material support: Choi. Study supervision: Chang-Hyun Lee, Chae-Heuck Lee.

\section{Correspondence}

Chang-Hyun Lee: Seoul National University Hospital, Seoul, Republic of Korea. imspinesurgeon@gmail.com. 